\title{
Observation and analysis of structural changes in fused silica by continuous irradiation with femtosecond laser light having an energy density below the laser-induced damage threshold
}

\author{
Wataru Nomura*1, Tadashi Kawazoe ${ }^{1}$, Takashi Yatsui ${ }^{1}$, Makoto Naruse ${ }^{2}$ \\ and Motoichi Ohtsu ${ }^{1}$
}

Open Access

\begin{abstract}
The laser-induced damage threshold (LIDT) is widely used as an index for evaluating an optical component's resistance to laser light. However, a degradation in the performance of an optical component is also caused by continuous irradiation with laser light having an energy density below the LIDT. Therefore, here we focused on the degradation in performance of an optical component caused by continuous irradiation with femtosecond laser light having a low energy density, i.e., laser-induced degradation. We performed an in situ observation and analysis of an increase in scattering light intensity in fused silica substrates. In experiments conducted using a pulsed laser with a wavelength of $800 \mathrm{~nm}$, a pulse width of $160 \mathrm{fs}$ and pulse repetition rate of $1 \mathrm{kHz}$, we found that the scattered light intensity increased starting from a specific accumulated fluence, namely, that the laser-induced degradation had a threshold. We evaluated the threshold fluence $F_{\mathrm{t}}$ as $6.27 \mathrm{~J} / \mathrm{cm}^{2}$ and $9.21 \mathrm{~J} / \mathrm{cm}^{2}$ for the fused silica substrates with surface roughnesses of $0.20 \mathrm{~nm}$ and $0.13 \mathrm{~nm}$ in $R_{\mathrm{a}}$ value, respectively, showing that the threshold decreased as the surface roughness increased. In addition, we found that the reflected light spectrum changed as degradation proceeded. We analyzed the details of the degradation by measuring instantaneous reflectance changes with a pump-probe method; we observed an increase in the generation probability of photogenerated carriers in a degraded silica substrate and a damaged silica substrate and observed a Raman signal originating from a specific molecular structure of silica. From these findings, we concluded that compositional changes in the molecular structure occurred during degradation due to femtosecond laser irradiation having an energy density below the LIDT.
\end{abstract}




\section{Introduction}

Since the invention of the laser, it has been widely known that damages occur at the surface or interior of optical components when irradiated with laser light having a high energy density. A lot of research has been conducted in the area of laser-induced damage. For example: mechanism for picosecond [1,2] and femtosecond (fs) laser [3,4], effect of surface geometry [5,6], damage threshold $[7,8]$, and so on. The laser-induced damage originates in photoionization of molecules caused by local electric fields generated at ultrafine structures in the substrates, such as indentations/protrusions in the surface or impurities in the interior. Recently, with advances made in the development of light sources, such as the trend towards lasers with higher power, shorter pulses, and shorter wavelengths, there has been a demand for the development of optical components having even higher damage resistance. Laser resistance is evaluated by an index called the laser-induced damage threshold (LIDT), which is measured by the 200-on-1 method according to ISO 11254-2 $[9,10]$, for example. With this method, 200 locations on a sample surface are irradiated with single shots of pulsed laser light having different energy densities. The presence/absence of damage sites due to the irradiation is visually checked, and the minimum energy density at which damages are found is defined as the LIDT. However, the practical problem is that the performance of an optical component, such as transmittance, reflectance, etc., is degraded by continuous irradiation with laser light having an energy density below the LIDT. The cause of such degradation in performance, namely, laser-induced degradation of the optical component, is not fully understood. In addition, the resistance cannot be evaluated by tests based on ISO 11254-2, and therefore, a technique for the quantitative evaluation of such degradation would be useful. Furthermore, if the relation between laser-induced degradation and surface roughness is revealed, it may contribute to the development of a surface polishing technology for optical components with higher resistance against not only laser-induced damage but also degradation. Moreover, the clarification of the mechanism and knowledge about the details of the laser-induced degradation may provide an opportunity to develop novel laser processing techniques. Thus, our study about laser-induced degradation is a contribution to the progress in nanotechnology.

Therefore, we performed in situ a quantitative detection of laser-induced degradation in flat fused silica substrates, serving as the target material. The continuous irradiation with fs pulsed laser light has an energy density below the LIDT. We also analyzed the origin of the laser-induced degradation. Section 1 in Results and Discussion describes experiments in which we continuously irradiated substrates with fs pulsed laser light having a low energy density below the LIDT and detected the laser-induced degradation by measuring the scattered light. In addition, by simultaneously measuring the reflected-light spectrum during this process, we observed spectral changes as the laser-induced degradation proceeded. Section 2 in Results and Discussion describes the measurement of instantaneous reflectance changes using a pump-probe method carried out to analyze the details of these spectral changes. From an increase in photo-generated carriers and the power spectrum of their relaxation lifetime, we found that compositional changes in the molecular structure occurred as laser-induced degradation proceeded.

\section{Results and Discussion}

\section{Evaluation of laser-induced degradation of} fused silica

\subsection{Surface roughness dependence of threshold fluence in laser-induced degradation}

First, to quantitatively observe laser-induced degradation due to continuous irradiation with a laser light having an energy density below the LIDT, we conducted an experiment in which we continuously irradiated a silica substrate with fs laser light and measured the relative increase in scattered light from the substrate surface. As measurement samples, we used highpurity fused silica flat substrates in which the $\mathrm{OH}$ impurity content was 0.8 ppm or less. We used two samples: sample A, which was prepared by planarizing a substrate using standard chemical-mechanical polishing (CMP) [11], and sample B, which was prepared by performing optical near-field etching on a substrate prepared under the same conditions as sample A. The samples $\mathrm{A}$ and $\mathrm{B}$ had a minimum average surfaces roughnesses $R_{\mathrm{a}}$ of $0.20 \mathrm{~nm}$ and $0.13 \mathrm{~nm}$, respectively [12]. Since we employed a continuous-wave laser with the wavelength of $532 \mathrm{~nm}$ and power of $2 \mathrm{~W}$ for optical near-field etching, the sample B did not have any laser-induced damage or degradation caused by this preparation. Optical near-field etching is a surface planarization technique for selectively removing only minute protrusions in the surface of a substrate, and can flatten the planer substrate polished by CMP. Thus, it is effective in reducing the drop in LIDT caused by electric field enhancement induced by the surface structure $[5,6]$. It has been reported that a dielectric multilayer mirror fabricated by using a silica substrate planarized by this technique exhibited an LIDT that was increased from $8.2 \mathrm{~J} / \mathrm{cm}^{2}$ to $14 \mathrm{~J} / \mathrm{cm}^{2}$ compared with the mirror before planarization processing, according to a 200-on-1 laser resistance test based on ISO 11254-2 [13].

Figure 1a shows the experimental set-up. For the light source, we used a regeneratively amplified fs Ti:sapphire laser (Coherent, Inc., Legend Elite F-1K) with a wavelength of $800 \mathrm{~nm}$, a power of $1.0 \mathrm{~W}$, a repetition frequency of $1 \mathrm{kHz}$, and a pulse width of $160 \mathrm{fs}$. The laser light was incident on the 
surface of the substrates at an incident angle of $45^{\circ}$ and with an energy density of $6.0 \mathrm{~mJ} / \mathrm{cm}^{2}$. This energy density is sufficiently lower than the LIDT of fused silica for the same wavelength and pulse width, namely, about $0.5 \mathrm{~J} / \mathrm{cm}^{2}[14,15]$. Scattered light from the samples was collected by a Si photodiode (Hamamatsu Photonics K.K., S3883) placed perpendicularly to the substrate, and lock-in detection was performed at the repetition frequency of the laser using a lock-in amplifier (Stanford Research Systems, Inc., SR830).

We evaluated the two silica substrates using the relative increase in the scattered light intensity $S, \Delta S(F)=(S(F)-S(0))$ / $S(0)$, versus the accumulated fluence $F\left(\mathrm{~J} / \mathrm{cm}^{2}\right)$ of the irradiated light. In the experiment, we continuously irradiated samples A and B with laser light having fluences up to $F=3.5 \times 10^{4} \mathrm{~J} / \mathrm{cm}^{2}$ and $8.8 \times 10^{4} \mathrm{~J} / \mathrm{cm}^{2}$, respectively, and after the experiment, we confirmed that there were no sites showing laser-induced damage in the surfaces of the samples by using an optical microscope. The results of measuring $\Delta S(F)$ for samples $\mathrm{A}$ and $\mathrm{B}, \Delta S_{\mathrm{A}}$ and $\Delta S_{\mathrm{B}}$, are shown by the black and red solid lines in Figure $1 \mathrm{~b}$, respectively. We observed a tendency for the scattered light to increase with continuing irradiation, showing that this method succeeded in quantitatively measuring the laserinduced degradation. In addition, for both $\Delta S_{\mathrm{A}}$ and $\Delta S_{\mathrm{B}}$, the scattered light intensity increased logarithmically from specific fluences, showing that there was a threshold fluence at which laser-induced degradation started. To determine this threshold fluence, $F_{\mathrm{t}}$, the regions where $\Delta S_{\mathrm{A}}$ and $\Delta S_{\mathrm{B}}$ rose were fitted with an approximation $g(F)=P \log (F)-Q$. The approximation curves are shown by the green and blue dotted lines in Figure $1 \mathrm{~b}$, where $g_{\mathrm{A}}(F)$ was defined over the range
$6.3 \times 10^{3} \mathrm{~J} / \mathrm{cm}^{2}<F<1.2 \times 10^{4} \mathrm{~J} / \mathrm{cm}^{2}$ for $\Delta S_{\mathrm{A}}$, and $g_{\mathrm{B}}(F)$ was defined over the range $F>9.2 \times 10^{3} \mathrm{~J} / \mathrm{cm}^{2}$ for $\Delta S_{\mathrm{B}}$. The constants $P$ and $Q$ are as shown in Table 1 . When $F_{\mathrm{t}}$ at which $g_{\mathrm{A}}(F)=0$ and $g_{\mathrm{B}}(F)=0$ is determined from these equations, for the samples $\mathrm{A}$ and $\mathrm{B}$, we calculated $F_{\mathrm{tA}}=6.3 \times 10^{3} \mathrm{~J} / \mathrm{cm}^{2}$ $\left(1.1 \times 10^{6}\right.$ shots $)$ and $F_{\mathrm{tB}}=9.2 \times 10^{3} \mathrm{~J} / \mathrm{cm}^{2}\left(1.5 \times 10^{6}\right.$ shots $)$, under the laser energy density of $6.0 \mathrm{~mJ} / \mathrm{cm}^{2}$. This result shows that the degradation starting threshold for sample B is 1.4-times higher than that for sample A. As described above, sample B was a substrate prepared by selectively removing only the ultrafine surface structure from the substrate of sample A, and the LIDT of a mirror using a substrate identical to this was improved by a factor of 1.7 [13]. Since the degradation starting threshold $F_{\mathrm{t}}$ and the LIDT improvement factor are in good agreement, this result indicates the laser-induced degradation occurs with a partly similar mechanism of laser-induced damage, namely multiphoton ionization caused by local electric fields generated at indentations or protrusions. Also, the constants $P$ which define the rate of increase of $g_{\mathrm{A}}(F)$ and $g_{\mathrm{B}}(F)$ are $P_{\mathrm{A}}=0.062$ and $P_{\mathrm{B}}=0.016$, differing by a factor of 3.9 , which shows that laser-induced degradation originating from the surface structure proceeds more rapidly.

Table 1: Constants $P$ and $Q$ for approximation curves $g_{\mathrm{A}}(F)$ and $g_{\mathrm{B}}(F)$.

\begin{tabular}{ccc} 
& $P$ & $Q$ \\
\hline$g_{\mathrm{A}}(F)$ & 0.062 & 0.24 \\
$g_{\mathrm{B}}(F)$ & 0.016 & 0.062
\end{tabular}

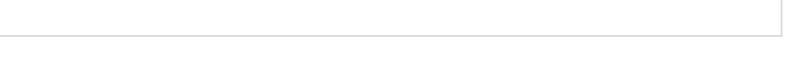

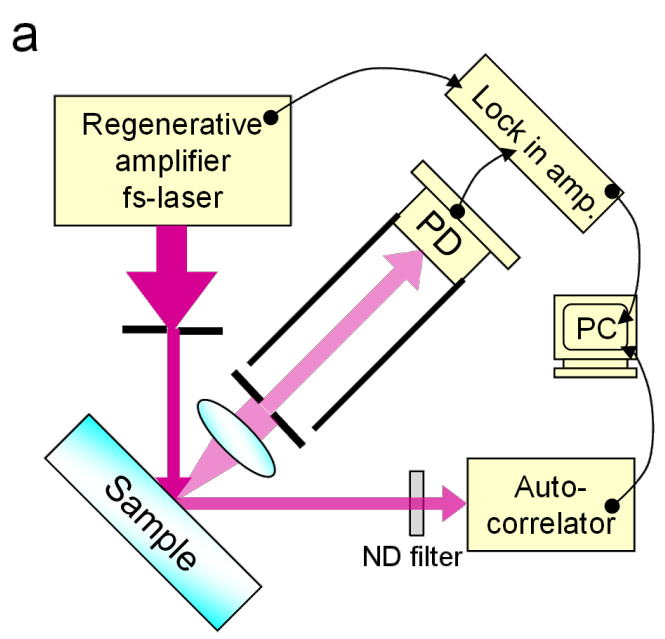

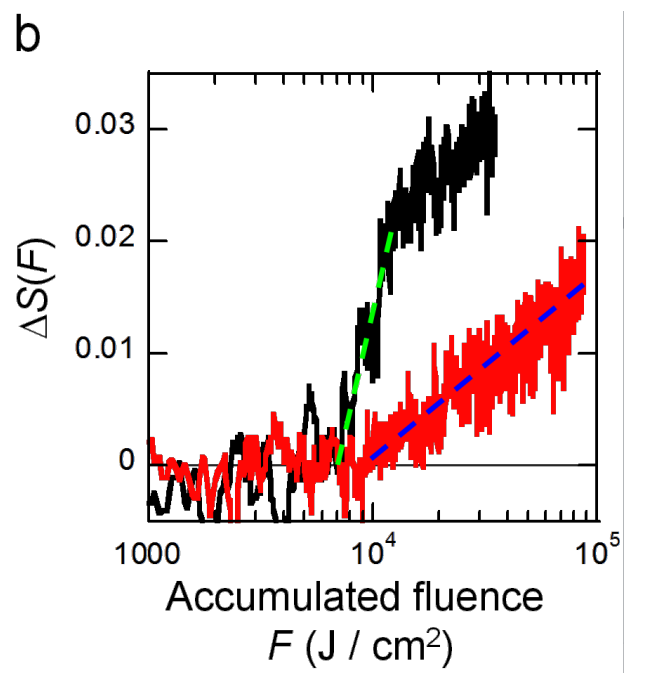

Figure 1: a) Schematic diagram of experimental set-up for measuring laser-induced degradation due to irradiation with energy density below the LIDT. b) Relative increase in scattered light intensity, $\Delta S$, versus accumulated fluence, $F$, for samples $A$ and $B$. Black and red solid lines show $\Delta S_{A}$ and $\Delta S_{B}$, respectively. The green and blue dotted lines show approximation curves $g_{A}(F)$ and $g_{B}(F)$ for regions where $\Delta S_{A}$ and $\Delta S_{B}$ rise. 


\subsection{In situ evaluation of spectral change in reflected} light

To analyze the laser-induced degradation, we evaluated the reflected light spectrum at the same time as the scattered light intensity measurement. As shown in Figure 1a, reflected light was introduced from the sample into an autocorrelator with a spectrometer function (APE GmbH, pulseCheck). We used a fused silica substrate identical to sample A and set the energy density of incident light on the substrate to $17 \mathrm{~mJ} / \mathrm{cm}^{2}$, which is below the LIDT. Spectral data was obtained at four fluences, namely, $F_{1}=5.1 \times 10^{3} \mathrm{~J} / \mathrm{cm}^{2}, F_{2}=1.9 \times 10^{5} \mathrm{~J} / \mathrm{cm}^{2}$, $F_{3}=5.9 \times 10^{5} \mathrm{~J} / \mathrm{cm}^{2}$, and $F_{4}=1.3 \times 10^{6} \mathrm{~J} / \mathrm{cm}^{2}$.

The solid black line in Figure 2a shows the wavenumber spectra of the reflected light, centered on the central wavelength of the laser $(800 \mathrm{~nm})$. Under all conditions, in addition to the central frequency of the laser, we observed the 2 nd peak on the longwavelength side. When these wavenumber spectra were fitted with the sum of two Gaussian functions, as shown by the solid red lines in Figure 2a, the center wavenumbers on the 2nd peaks $K$ were determined to be $K_{1}=84 \mathrm{~cm}^{-1}, K_{2}=86 \mathrm{~cm}^{-1}$, $K_{3}=92 \mathrm{~cm}^{-1}$, and $K_{4}=94 \mathrm{~cm}^{-1}$ for the fluences $F_{1}$ to $F_{4}$, respectively. The relative increase in the scattered light intensity, $\Delta S$, and $K$ versus the irradiated fluence, $F$, are plotted in the black solid line and the red squares in Figure $2 b$, respectively. The monotonically increasing trends with respect to $F$ agreed well in both $\Delta S$ and $K$, showing that the 2nd peak of the spec- trum shifted to lower energy side as the degradation proceeded. We estimated the origin of the 2 nd peaks were Raman signals of degraded silica and this result represented the compositional changes in the molecular proceeds with the laser-induced degradation proceeded.

\section{Analysis of degradation via pump-probe method \\ 2.1 Time-resolved measurement of reflectance}

To analyze in detail the spectral changes occurring with laserinduced degradation due to continuous irradiation with laser light having an energy density below the LIDT, as confirmed in the previous section, we conducted an experiment to evaluate the time-resolved changes in reflectance via a pump-probe method. The experimental setup is shown in Figure 3. The laser used was a mode-locked Ti:sapphire laser (Coherent, Inc., Mira Optima 900-D) with a wavelength of $750 \mathrm{~nm}$, a pulse width of $90 \mathrm{fs}$, and a repetition frequency of $80 \mathrm{MHz}$. Using a beamsplitter, the laser light was split with an intensity ratio of reflected light to transmitted light of 1:19. As pump light, the transmitted light was made perpendicularly incident on the sample surface via an optical delay and a chopper with a frequency of $2 \mathrm{kHz}$. P-polarized probe light, with a polarization orthogonal to that of the pump light, was incident on the substrate at an angle of incidence of about $70^{\circ}$, and the reflected probe light was received by a $\mathrm{Si}$ photodiode (Hamamatsu Photonics K.K., S3883). The received signal was detected with a

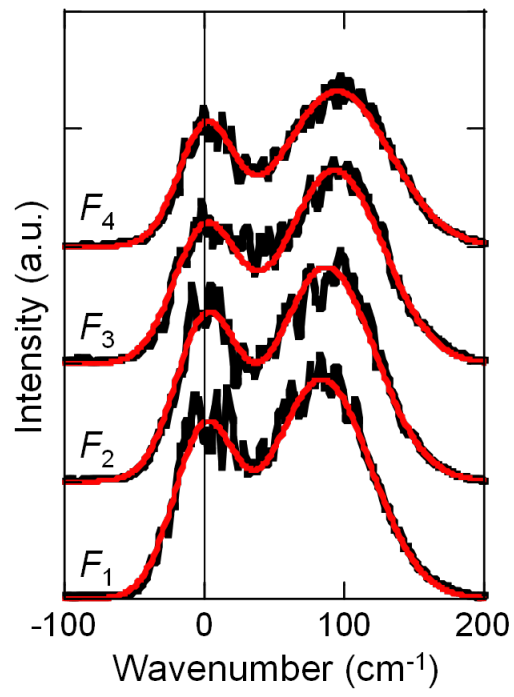

b

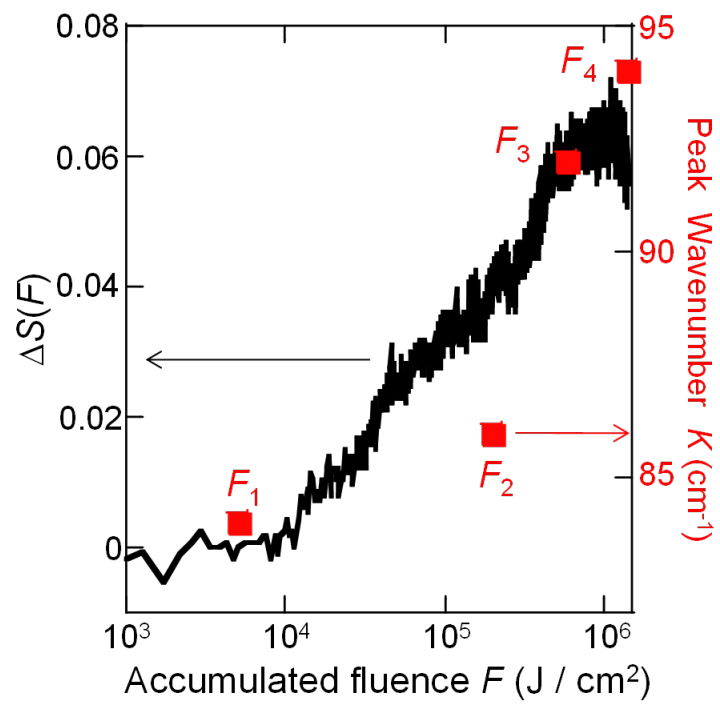

Figure 2: a) Wavenumber spectra of reflected light from silica substrate for accumulated fluences $F_{1}$ to $F_{4}$, where $F_{1}=5.1 \times 10^{3} \mathrm{~J} / \mathrm{cm}^{2}$, $F_{2}=1.9 \times 10^{5} \mathrm{~J} / \mathrm{cm}^{2}, F_{3}=5.9 \times 10^{5} \mathrm{~J} / \mathrm{cm}^{2}$, and $F_{4}=1.3 \times 10^{6} \mathrm{~J} / \mathrm{cm}^{2}$. The black and red solid lines indicate the measured values and approximation curves given by the sum of two Gaussian functions, respectively. The vertical axis shows arbitrary units and the baselines of $F_{1}$ to $F_{4}$ are essentially equivalent. b) Dependence of relative increase in scattered light intensity, $\Delta S$ (black line), and peak wavenumber in reflected light spectra $K$ (red squares) on accumulated fluence, $F$. 


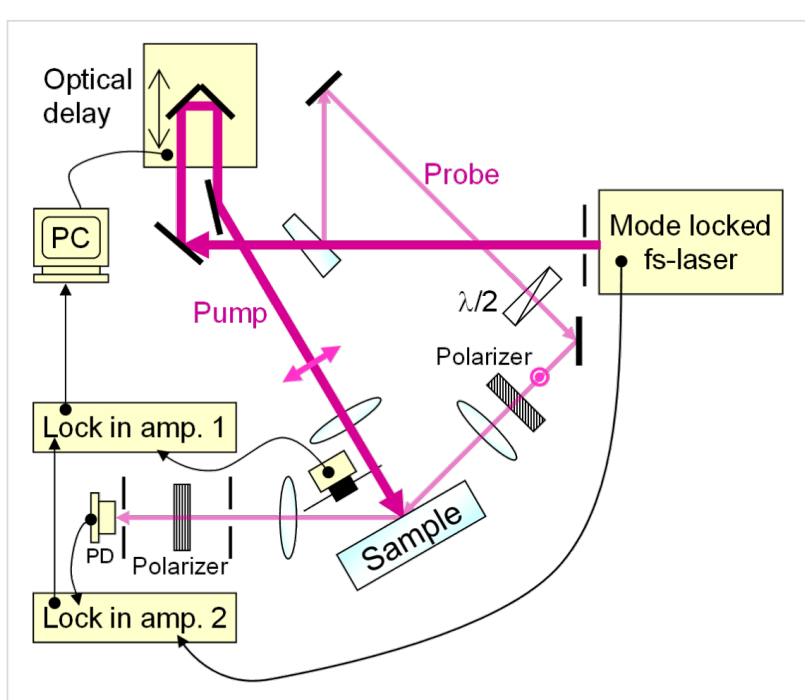

Figure 3: Schematic diagram of pump-probe experimental set-up.

double lock-in detection using two lock-in amplifiers (Stanford Research Systems, Inc., SR844 and SR830) based on the repetition frequency of the laser pulses and the frequency of the chopper. The laser light used as the pump light in this experiment had an energy density of $1.6 \mu \mathrm{J} / \mathrm{cm}^{2}$, and no increase in light scattering was observed even when the silica substrate was continuously irradiated with this laser light with a accumulated fluence exceeding $1.0 \times 10^{7} \mathrm{~J} / \mathrm{cm}^{2}$, thus confirming that the progression of laser-induced degradation could be ignored in this pump-probe experiment. We performed measurements on three samples, which were all high-purity fused silica substrates polished by CMP, identical to sample A: Sample C, on which no laser was irradiated; Sample D, which showed laser-induced degradation by being irradiated with the regeneratively amplified fs laser with a wavelength of $800 \mathrm{~nm}$, an energy density of
$17 \mathrm{~mJ} / \mathrm{cm}^{2}$, a repetition frequency of $1 \mathrm{kHz}$ and a pulse width of $160 \mathrm{fs}$ to an accumulated fluence of $3.5 \times 10^{4} \mathrm{~J} / \mathrm{cm}^{2}$, namely $2.1 \times 10^{7}$ shots; and Sample E, in which laser-induced damage visually recognizable as damage sites was caused by 1000 shots of irradiation with an energy density of $100 \mathrm{~mJ} / \mathrm{cm}^{2}$ using the same fs laser.

In the evaluation, we used a relative change $\Delta R=\left(R-R_{0}\right) / \mathrm{R}_{0}$, where $R_{0}$ is the reflected light intensity in the case where only the probe light was radiated, and $R$ is the reflected light intensity obtained in the pump-probe experiment. The measurement results $\Delta R_{\mathrm{C}}, \Delta R_{\mathrm{D}}$, and $\Delta R_{\mathrm{E}}$ for samples $\mathrm{C}, \mathrm{D}$, and $\mathrm{E}$ are plotted in Figure $4 \mathrm{a}$ as black squares, blue circles, and red triangles, respectively. The horizontal axis of the graph is the time delay, $t$, of the probe light relative to the pump light, where the peak of $\Delta R_{\mathrm{C}}$ is taken as $t=0 . \Delta R_{\mathrm{C}}$ is well-approximated by $\Delta R_{\mathrm{fit}}=5.5 \operatorname{sech}^{2}\left(t /\left(9 \times 10^{-14}\right)\right)$, which is shown by the green solid line in Figure $4 \mathrm{a}$, and nonlinear polarization reflecting the pulse width of the laser was observed. In contrast, $\Delta R_{\mathrm{D}}$, and $\Delta R_{\mathrm{E}}$ showed temporal broadenings of about several $100 \mathrm{fs}$. Because these decay times are close to the photogenerated carrier lifetime of silica $150 \mathrm{fs}[16,17]$, it is assumed the temporal changes in the reflectance were originated from the photogenerated carrier in samples D and E. In other words, in Samples D and E, we concluded that the generation probability of photogenerated carriers was higher than in Sample $\mathrm{C}$ because electric field enhancement occurred due to laser-induced degradation and laser-induced damage.

\subsection{Spectrum analysis}

To analyze this in more detail, we obtained the power spectrum by subtracting the nonlinear polarization component represented by $\Delta R_{\mathrm{fit}}$ from $\Delta R_{\mathrm{D}}$, and $\Delta R_{\mathrm{E}}$. The results are shown by a

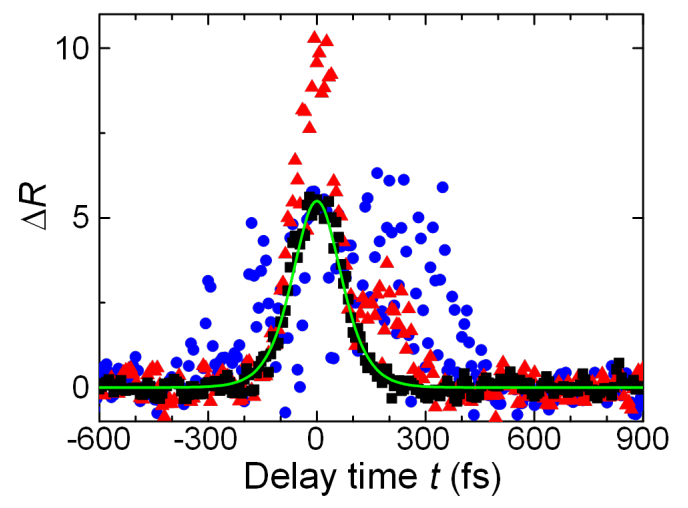

b

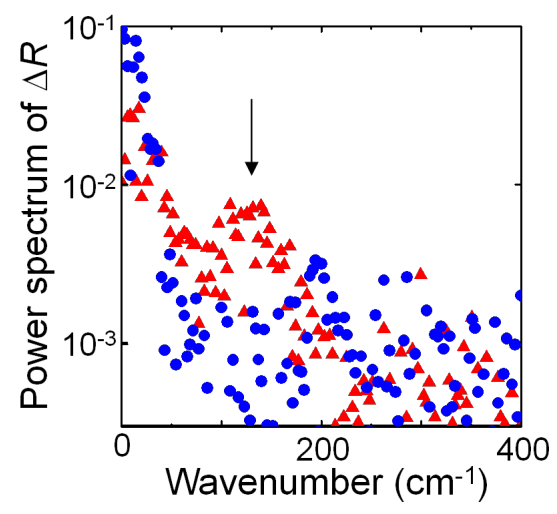

Figure 4: a) Instantaneous relative increase in reflected light intensity, $\Delta R$, measured by pump-probe method. Black squares, blue circles, and red triangles indicate measurement results for samples $\mathrm{C}, \mathrm{D}$, and $\mathrm{E}$, respectively. The solid green line indicates the approximation curve $\Delta R_{\mathrm{fit}}=5.5 \mathrm{sech}^{2}\left(t /\left(9 \times 10^{-14}\right)\right)$ for $\Delta R_{\mathrm{C}}$. b) Power spectrum obtained by subtracting the $\Delta R_{\mathrm{fit}}$ component from $\Delta R_{\mathrm{D}}$, and $\Delta R_{\mathrm{E}}$. The blue circles and red triangles indicate the analysis results for $\Delta R_{\mathrm{D}}$, and $\Delta R_{\mathrm{E}}$, respectively. The arrow indicates $128 \mathrm{~cm}^{-1}$. 
the blue circles and red triangles in Figure 4b, respectively. For sample D, a signal having a weak peak close to $195 \mathrm{~cm}^{-1}$ was obtained, whereas for sample E, a broad peak around $128 \mathrm{~cm}^{-1}$ was found, thus giving different signals. The peak at $128 \mathrm{~cm}^{-1}$ was consistent with the Raman signal of $\alpha$-quartz, which is formed of six-membered rings of $\mathrm{Si}$ and $\mathrm{O}$ [18]. It has been reported that the Raman signal from silica with a specific molecular structure is increased by irradiation with fs laser light having an energy density above the LIDT [19], and sample E is also thought to be mainly formed of six-membered rings in a similar fashion. Although there is no molecular structure of silica that corresponds to the peak for sample D, the result indicates that compositional changes occur due to laser-induced degradation. As we found in section 1.1, the laser-induced degradation originates in multiphoton ionization. The laser-induced damage by fs laser occurs by multiphoton ionization and consequent avalanche ionization $[3,4]$. Since there are no breakdowns, it is estimated that avalanche ionization does not occur in the laser-induced degradation due to its low energy density. While the carriers generated by just multiphoton ionization are not enough to cause breakdown, they change amorphous silica to a more stable molecular structure. As also indicated in the results in section 1.2, the spectrum shifted, namely the compositional change carried on as laser-induced degradation proceeds. It provides nanoscale nonuniformity of the refractive index which increases scattering of light, decreases transmittance, and accelerates the progress of the laser-induced degradation.

\section{Conclusion}

In this paper, we focused on the degradation in performance of optical components due to continuous irradiation with laser light having an energy density below the LIDT, i.e., laserinduced degradation. We examined the degradation of fused silica substrates in response to fs laser irradiation and performed an in situ observation of the laser-induced degradation, as well as an analysis of the underlying mechanism. By monitoring the scattered light intensity versus the accumulated irradiation fluence, we succeeded in quantitatively detecting the laserinduced degradation. We confirmed that the total irradiation threshold at which laser-induced degradation starts changed depending on the size of the ultrafine structure in the surface. In addition, we also found that the spectrum of reflected light shifted as the laser-induced degradation proceeded. By analyzing the details of this behavior with a pump-probe method, we observed an increase in photogenerated carriers, and from the power spectrum, we obtained results indicating that the molecular structure of the silica undergoes compositional changes due to the laser-induced degradation.

From the findings we obtained in this study, it can be concluded that, to increase the resistance of a substrate to laser-induced de- gradation, it is effective to improve the substrate's flatness, similarly to the case of the LIDT. In addition, because it is possible to estimate the molecular structure formed by the laserinduced degradation from the spectral information, there is a possibility of achieving more effective measures against laserinduced degradation. The measurement method employed here for the fs laser light and the flat fused silica substrates can also be applied to other types of laser and optical components and is expected to contribute to the development of optical components having high resistance to laser-induced degradation in the future.

\section{Acknowledgements}

The authors would like to thank Prof. Dr. M. Haraguchi of The University of Tokushima, Mr. Y. Tabata and Mr. K. Hirata of SIGMA KOKI Co., Ltd., for helpful discussions. This work was partially supported by the New Energy and Industrial Technology Development Organization (NEDO) under the program of Strategic Development on Rationalization Technology using Energy and under the program of Comprehensive Activity for Personnel Training and Industry-Academia Collaboration based on the NEDO project.

\section{References}

1. Bliss, E. S. Opto-electronics (London) 1971, 3, 99-108. doi:10.1007/BF01424087

2. Wood, R. M. Laser Damage in Optical Materials; Hilger: Boston, 2002.

3. Schaffer, C. B.; Brodeur, A.; Mazur, E. Meas. Sci. Technol. 2001, 12, 1784-1794. doi:10.1088/0957-0233/12/11/305

4. Mao, S. S.; Quéré, F.; Guizard, S.; Mao, X.; Russo, R. E.; Petite, G.; Martin, P. Appl. Phys. A 2004, 79, 1695-1709. doi:10.1007/s00339-004-2684-0

5. Génin, F. Y.; Salleo, A.; Pistor, T. V.; Chase, L. L. J. Opt. Soc. Am. A 2001, 18, 2607-2616. doi:10.1364/JOSAA.18.002607

6. Bloembergen, N. Appl. Opt. 1973, 12, 661-664. doi:10.1364/AO.12.000661

7. Stuart, B. C.; Feit, M. D.; Herman, S.; Rubenchik, A. M.; Shore, B. W.; Perry, M. D. Phys. Rev. B 1996, 53, 1749-1761. doi:10.1103/PhysRevB.53.1749

8. Jia, T. Q.; Li, R. X.; Liu, Z.; Xu, Z. Z. Appl. Phys. A 2002, 74, 503-507. doi:10.1007/s003390100903

9. Becker, J.; Bernhardt, A. Proc. SPIE 1994, 2114, 703-713. doi:10.1117/12.180881

10. ISO 11254-2, Test methods for laser induced damage threshold of optical surfaces. Part 2: S on 1 test, International Organization for Standardization. 2001.

11. Cook, L. M. J. Non-Cryst. Solids 1990, 120, 152-171. doi:10.1016/0022-3093(90)90200-6

12. Yatsui, T.; Hirata, K.; Nomura, W.; Tabata, Y.; Ohtsu, M. Appl. Phys. B 2008, 93, 55-57. doi:10.1007/s00340-008-3142-z

13. Hirata, K. Proc. SPIE 2011, 7921, 79210M. doi:10.1117/12.875808

14. Miura, K.; Qiu, J.; Inouye, H.; Mitsuyu, T.; Hirao, K. Appl. Phys. Lett. 1997, 71, 3329-3331. doi:10.1063/1.120327 
15. Sudrie, L.; Couairon, A.; Franco, M.; Lamouroux, B.; Prade, B.; Tzortzakis, S.; Mysyrowicz, A. Phys. Rev. Lett. 2002, 89, 186601. doi:10.1103/PhysRevLett.89.186601

16. Audebert, P.; Daguzan, P.; Dos Santos, A.; Gauthier, J. C.;

Geindre, J. P.; Guizard, S.; Hamoniaux, G.; Krastev, K.; Martin, P.; Petite, G.; Antonetti, A. Phys. Rev. Lett. 1994, 73, 1990-1993. doi:10.1103/PhysRevLett.73.1990

17. Saeta, P. N.; Greene, B. I. Phys. Rev. Lett. 1993, 70, 3588-3591. doi:10.1103/PhysRevLett.70.3588

18. Bates, J. B. J. Chem. Phys. 1972, 56, 1910-1917. doi:10.1063/1.1677474

19. Chan, J. W.; Huser, T.; Risbud, S.; Krol, D. M. Opt. Lett. 2001, 26, 1726-1728. doi:10.1364/OL.26.001726

\section{License and Terms}

This is an Open Access article under the terms of the Creative Commons Attribution License

(http://creativecommons.org/licenses/by/2.0), which permits unrestricted use, distribution, and reproduction in any medium, provided the original work is properly cited.

The license is subject to the Beilstein Journal of

Nanotechnology terms and conditions:

(http://www.beilstein-journals.org/bjnano)

The definitive version of this article is the electronic one which can be found at: doi:10.3762/bjnano.5.146 